\title{
Over break control in underground mines
}

\author{
Abstract \\ Blasting is an integral part of hard rock mining and remains the most cost effective \\ method of hard rock fragmentation. However, the costs associated with blast damage \\ in terms of safety and productivity of mines are becoming a serious concern. In order \\ to understand the mysterious nature of blast damage prediction and control, field \\ work involved the small scale blasting of physical models and monitoring of blasts \\ in operating hard rock mines. Understanding the factors affecting blast damage is an \\ important step for blast damage control and the refinement of blasting practice and \\ design for damage minimization. Small scale blasting and the monitoring of field \\ blasting practice provided a better understanding of the performance of the blasts in \\ general and identified which aspects of blasting had a significant impact upon over \\ break control. In the light of the field work, following aspects have been discussed to \\ minimize over break in underground excavations. \\ a. Drilling and blasting practice \\ b. Explosive characteristics and distribution \\ c. Explosive products for perimeter control \\ d. Blast design \\ e. Relevance of rock mass characterization to blasting results \\ In addition, based upon the review of the case histories and work done by the author \\ at different sites, a criterion for minor and major blast damage has been proposed. \\ Keywords: Blast damage control, rock mines, excavation process, structural \\ stability, blast holes, rock mass, poor fragmentation, blast design, Weathering of \\ rocks, Bore hole pressure, drilling and loading, drilling and blasting crew, excavation \\ process, sub-standard blasting results, detonators
}

Volume 4 Issue 5 - 2018

\author{
S Paul Singh \\ Laurentian University, Canada
}

Correspondence: S Paul Singh, Laurentian University, Canada, Emailssingh@laurentian.ca

Received: July 24, 20I8 | Published: December 31, 2018

\section{Introduction}

Blasting still remains the most cost effective method of hard rock fragmentation but it often inflicts damage to the surrounding rock. Blast damage to the periphery of an excavation is directly related to the level of stress experienced by the rock and its pre-blasting condition. Blast damage control reduces scaling time and dilution, allows easier and effective support installation and improves safety of the working environment. In fact, control of blast damage to mining excavations has become an economic imperative. In order to understand the mysterious nature of blast damage prediction and control, field work involved the small scale blasting of physical models, pipe tests and monitoring the effect of blasting in operating mines.

\section{What is blast damage?}

Blast damage is a change in rock properties which degrades its performance and behaviour. It causes deterioration in rock mass due to the presence of newly generated or extended fractures or opening and shearing along cracks and joints. Blast damage not only includes the breaking and rupturing of rock beyond the designed limits of excavation but also an unwanted loosening, dislocation and disturbance to the rock mass. Blast damage determines an important link between the excavation process and structural stability of the rock mass. Basically, it can be stated that the conventional blasting causes cracks and fractures in rock, which is manifested as blast damage Figure 1. In order to control blast damage, it is required that the blasting process produces the macro-cracks between the contour holes and eliminates micro-cracks in the remaining rock. ${ }^{1}$ A question was often raised whether the cracks are first generated around or between the contour holes. In order to answer this question, small scale blasting experiments were conducted on concrete samples. It was observed that, if the hole spacing and charge concentration is optimum, initial cracks are produced between the blast holes. In cases, where, hole spacing and charge concentration are very low or high, then cracks will develop almost simultaneously around as well as in the direction of the neighbouring holes.

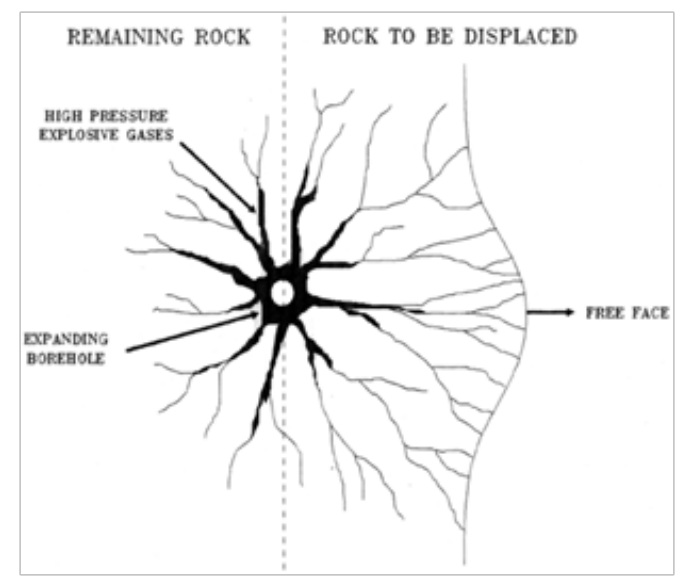

Figure I Typical crack formation and propagation around a blasthole. 


\section{Factors affecting blast damage}

The rock mass is often blamed for poor blasting results, even if the fault lies with the blast design or blasting practice. Therefore, in order to refine the blasting practice, factors influencing blast damage should be critically analysed to judiciously decide the main cause of sub-standard blasting results. Factors influencing blast damage can be divided into following three categories.

\section{Rock mass quality}

Rock mass is a heterogeneous and anisotropic medium, a fact often ignored during blast design. The use of standard blast designs, without any consideration to the variations in the rock mass conditions, results in blast damage and poor fragmentation. Rock mass features cannot be changed but their knowledge facilitates the judicious selection of the explosive characteristics and the blast design parameters to obtain optimum results. The following rock mass characteristics largely control the blast damage and blasting performance..$^{2-4}$

Strength and density of rocks

1. Number of joints

2. Aperture, frequency and orientation of joints

3. Joint filling and joint conditions

4. Layers or foliation

5. Weathering of rocks

6. Hydro-geological conditions

7. Rock quality designation

8. Rock mass rating.

\section{Explosive characteristics and distribution}

Explosive products release their energy and interact with rocks in different ways due to the difference in their constituents and reaction characteristics. Following parameters warrant due consideration during explosive selection and loading:
A. Velocity of detonation (VOD)
B. Bore hole pressure
C. Powder factor

\section{Blast design and execution}

It has been observed that the blast damage often extends beyond the perimeter holes. If the damage has already occurred by the earlier firing charges, then it is too late to rectify the problem by using perimeter control techniques. If the entire blast has been properly designed, drilled and loaded then perimeter control techniques can facilitate effective control on blast damage. The following factors can have significant impact on blast damage.
a) Drill hole deviation
b) Blast hole parameters
c) Cut design and blasting
d) Baby arch (Buffer) holes
e) Perimeter hole pattern and amount of explosive

f) Delay time and sequencing of holes.

\section{Blast damage control}

The damage to the rock mass caused by blasting can have a significant impact on the economy, productivity and safety of underground excavations. With the growing economic pressures faced by mining engineers, blast damage control programs are demanding more attention than before.

\section{Drilling and blasting practice}

It is important to remember that the blast damage control starts with the first hole to be marked and drilled.

\section{Drilling and blasting crew}

Drilling and blasting process is ten percent design and ninety percent implementation of the design and practice. This ninety percent component is solely in the hands of the drilling and blasting crew. Therefore, the crew should be well trained, motivated and understand the benefits of perimeter control. The key to the success of a blast damage control program lies in its acceptance by the crew. This depends upon the following. ${ }^{5}$

Effect on bonus

a. Degree of deviation from the current practice

b. Effect on safety and working environment

c. Nature and type of the technique

d. Hole loading time

e. Consistent, measurable and observable results

f. Management's commitment and support to the practice

\section{Preparation of drilling face}

Face preparation is a critical component of the blasting cycle. Before marking and drilling, the face should be scaled and washed. This will ensure that loose material is removed and all bootlegs are cleaned out and properly flagged. This will facilitate easier, safer and accurate drilling and loading.

\section{Face mark-up}

Grade and reference line should be painted. All grid lines and holes should be properly marked. Typical mark up of the face is shown in Figure 2 .

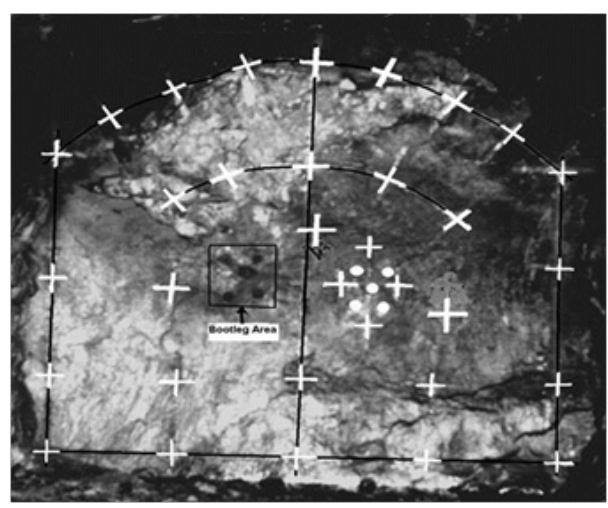

Figure 2 Typical mark up of the face before drilling 


\section{Accurate drilling}

The importance of drilling accuracy cannot be overstated when considering perimeter control. Any errors during survey, collaring or alignment will be magnified in direct proportion to the depth of the holes. Inaccurate drilling will not allow desirable advance of the round and will cause more damage. Time and attention spent for accurate surveying, marking, collaring and drilling of holes contributes towards minimum damage. Following Suggestions should be kept in mind while drilling the round to minimize damage. Ensure that the designed pattern is properly followed:

1. It is better to keep the face square and don't drill holes to full depth on an uneven face.

2. When drilling cut, use a guide pole to line up rest of the cut and helpers. A single boom jumbo is more difficult to align than a two boom jumbo, where the booms can be used to align each other.

3. Ensure proper burden and spacing for contour and buffer holes.

4. Improper lookout angle can result in saw-tooth back and excessive over break. Use extra caution on corner holes.

5. All holes including reamers must be blown.

\section{Loading of holes}

After drilling accuracy, explosive loading practices have next most control on blast damage. Before charging, all holes should be cleaned to remove water and drill cuttings. Assign delays to ensure the sequential development of free faces. Ensure all detonators are tied in and detonating cord is properly secured into the shock tube connectors. Make sure that the Initiating cap for the detonating cord is couple of meters away from the face.

\section{Explosive characteristics and distribution}

Explosive characteristics play a vital role in producing blast damage. The difference in damage from different explosives is attributed to the difference in the

1. Energy release rate which is a function of the difference in the velocities of detonation.

2. Magnitude and rate of bore hole pressurization.

3. Energy transmission to the rock

4. Strain rate

\section{Bore hole pressure and velocity of detonation}

The bore hole pressure of an explosive is related to its density and velocity of detonation. In fact, higher the VOD of an explosive, higher will be its bore hole pressure. But we find in the blasting literature that higher bore hole pressure produces more damage whereas in Figure 3 , it has been displayed that higher the VOD of an explosive lower is the damage. It appears contradictory, but it is true due to the reason that high VOD explosives are decoupled and yield higher shock energy and less gas energy. High VOD explosives have low pulse duration and provide less time for crack growth. Explosives with high detonation velocity, if coupled, produce more cracks close to the blast hole walls. Due to the decoupling effect, shock energy is dissipated in a less harmful manner and the longer acting gas energy is more important from the damage point of view. Shock and gas energies

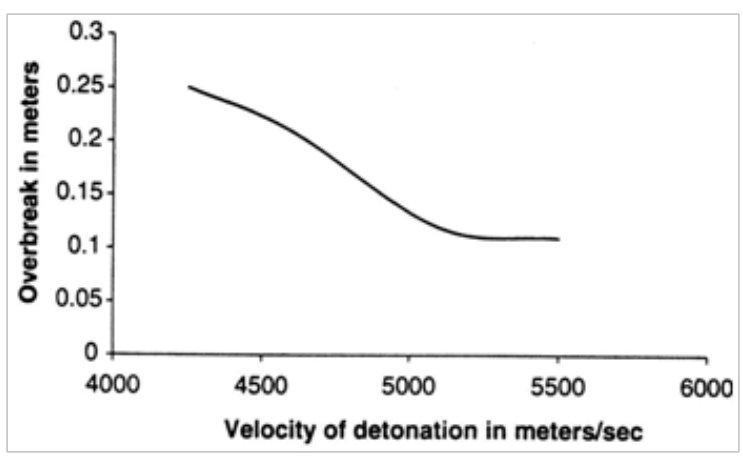

Figure 3 Velocity of detonation of explosives V/s over break.

The VOD of an explosive determines its energy partitioning characteristics. In the case of high VOD explosives the total energy is split more in favour of shock energy and vice versa. It is clear by now that two basic energy sources for blasting are shock and gas energies. It is imperative to unmask the damage causing potential of these two energy sources.

Maximum shock energy is transmitted to the rock when the contact area between the explosive and the rock is maximum. That is why; shock energy is less effective in producing damage when decoupled charges are employed.

1. During pipe tests, it was found that the VOD of the explosive was higher in the center than the sides. Therefore, it appears, as the shock front proceeds it is becoming somewhat conical.

2. The shock energy is applied to the borehole wall in the form of a narrow circle of pressure, which proceeds along the explosive column in the direction of detonation front. If this circle of pressure was stationary, it would act normal to the wall. Due to the forward motion of the circle of pressure, the shock energy is directed at an angle to the side walls, thus reducing its effectiveness. Therefore, shock energy is less effective in producing damage proportional to its magnitude.

3. The duration of the gas pressure effect is so long that it can supply enough energy to the crack propagation process. But the effective use of this energy depends upon the rock being preconditioned by the shock energy. The jointed rock mass in the mines is also considered preconditioned, but joints may promote or arrest the crack propagation process depending upon their orientation.

4. The shock energy causes fracture at the bore hole wall and initiates micro fractures during interaction with the discontinuities. This transient pressure pulse outruns the fracture propagation process because the propagation velocity of the pulse is approximately three to five times the crack propagation velocity. Therefore, shock energy does not contribute to the extension of longer cracks.

5. It was observed during the study that crushed material caused by the shock energy sometimes sticks along the half barrel but in small patches, still giving enough space for the high pressure gases to enter the radial cracks and extend them.

6. The shock wave damaged surface was observed to be smooth and hard whereas the gas pressure damage surface was undulating, fragile and exhibited a charred appearance. 


\section{Why decoupled explosives?}

An explosive is decoupled when its diameter is less than the diameter of the blast hole. Decoupling is done to reduce the charge concentration in the blast hole and minimize stresses exerted on the borehole walls. When a charge is decoupled following effects are occurring: hole.

There is a reduction in the linear density of the explosive in a bore

Direct transmission of the detonation shock wave to the rock mass is prevented if there is no water present in the hole to fill the annulus.

a) The bore hole wall is pressurized with a lower initial gas pressure because the volume of the bore hole is higher than the explosive charge.

b) Decoupling may cause a potential change in the detonation characteristics of an explosive.

c) The gap between the explosive and the borehole decreases the peak of the shock wave and weakens the loading strain rate. As a result of that crushed zone and the length \& intensity of cracks around the blast hole reduces. ${ }^{6}$

d) Explosive products for perimeter control

e) The selection of an appropriate explosive and its proper use are critical to an effective blast. The following explosive products are used in Canadian underground mines for perimeter control:Semigelatine dynamite cartridges with couplers

The explosive is packaged in rigid two feet cardboard tubes and couplers. The diameter of these cartridges is about $19 \mathrm{~mm}$. The results depend upon the compatibility between the coupling ratio and the rock mass characteristics. In moderately jointed quartzite with a $32 \mathrm{~mm}$ diameter hole it produced some damage but in a $38 \mathrm{~mm}$ and $45 \mathrm{~mm}$ diameter holes in the same rock it gave good results.

Water-gel and emulsions in different forms:

1. Decoupled cartridges

2. Decoupled continuous (Sausages) length products in plastic

3. Decoupled continuous length ( Sausages) products with detonating cord in the center

\section{String loading of bulk products}

In general, they produce good results. However, these products are expensive and it takes more time to load as compared to ANFO. Sometimes decoupled products are found in the muck pile after blasting. Some Miners complained of intact collars left after the blast and difficulties in pulling the toes. It was experienced that continuous length sausages were harder to load than long rigid cartridges. Bubble sensitized emulsions and water-gels are more susceptible to channel effect and dead pressing.

ANFO in different forms ANFO, if used alone, is brutal to the perimeter of an excavation. Therefore, for perimeter control,

ANFO is used in following different forms:

A. Diluted ANFO (Different mixtures of ANFO and inert material)

B. ANFO traced with low strength detonating cord (Tracer blasting)

\section{Back blowing of ANFO}

\section{ANFO/Swedish spoon}

E. Decoupled ANFO in plastic liners

Several miners complained about the excessive fines dispersed in the air while loading diluted ANFO pneumatically. It produces good results if the dilution is compatible with the rock conditions. Tracer blasting is commonly used in Canadian underground mines for perimeter control. This involves placing a low strength detonating cord along the wall of a blasthole before charging the main column of ANFO. Although, it is not a panacea for overbreak control in all mining situations, it produces good results if properly applied. The mechanism of tracer blasting has been explained in. ${ }^{7}$ Back blowing of ANFO and ANFO spoon were used in some mines but are not very popular. They basically reduce the quantity of ANFO loaded in the holes. Some people believe, it produces low dynamic impact because of the reduced quantity of ANFO and deflagration of ANFO instead of detonation. Miners don't like to use decoupled ANFO in plastic liners because it requires higher loading time but it is a good alternative in wet holes.

\section{High strength detonating cord}

This produces good results but it does not meet the fume class requirements in Canadian underground mines. It is expensive, needs higher loading time and difficult to lock it in the hole. There is difficulty in pulling toes and sometimes it is found in the muck pile. The comparison of blast damage for different explosive products has been shown in figure 4 . Half cast factor has been used as a measure of blast damage. This is length of the half barrels after the blast expressed as a percentage of the initial length of the blast holes. Higher the value of the half cast factor, lower is the blast damage.

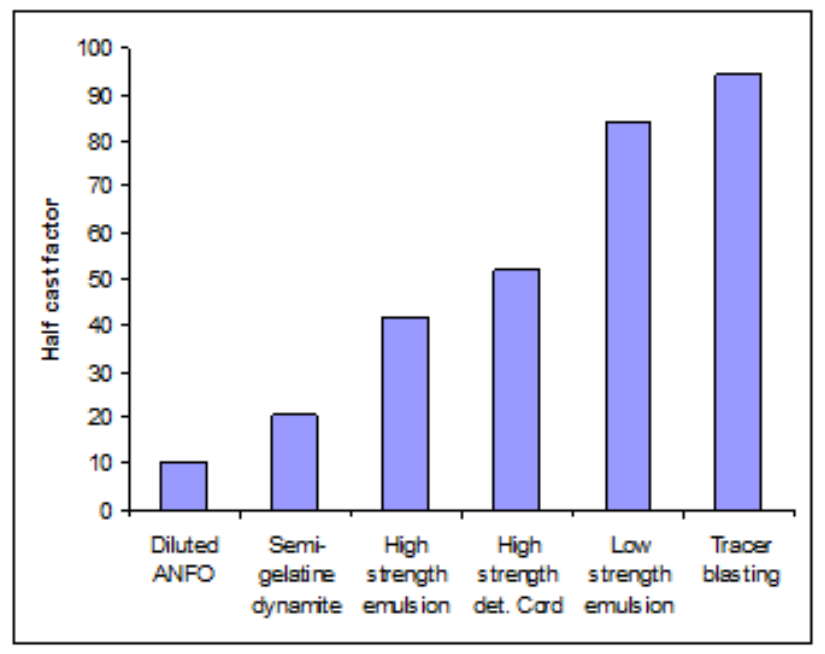

Figure 4 Half cast factors for different types of explosives.

\section{Blast design}

\section{Cut and blast damage}

A tunnel or drift blast starts with a cut because it provides a free face to which the remainder of the round may break. It is the most critical part of the blast design because the round cannot pull to the designed depth unless the cut comes out as planned. The events associated with the pulling of the cut are so remote from the perimeter 
that there is a tendency to believe that blasting of cut holes has no significant effect on blast damage. But that is not true. If progressive relief is not achieved in the cut area, then later firing charges will be over confined thus resulting in higher magnitude of ground vibrations and significant damage. Minimum blast damage requires that each hole fragments and displaces its burden with a reasonable ease. ${ }^{8}$ Damage to the drift face from cut blasting has been shown in Figure 5. The following suggestions during cut blasting are made to minimize damage to the perimeter:

a. The first four holes in the cut should be fired on separate and sufficient delays to provide progressive relief of burden and to avoid the superposition of ground vibrations.

b. The earliest firing charge should be closest to the larger relief area, distant from the perimeter holes and with its major pressure acting sideways.

c. The second charge should fire horizontally towards the first hole

d. Out of the cut holes firing vertically, the top one should go first

e. All other charges in the cut and cut helper area should be fired to meet the requirements of increasing free face effectiveness.

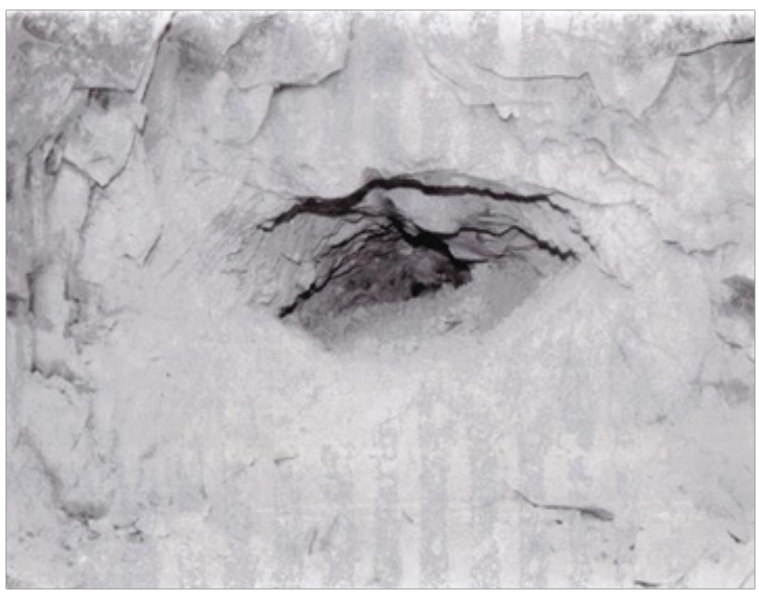

Figure 5 Damage to the drift face from cut blasting.

\section{Buffer holes}

These are also called first-row-in or baby arch holes and are adjacent to the perimeter holes. The care in drilling and charging of these holes is important but often overlooked. Positioning them close to the contour of the excavation and overcharging results in damage to the perimeter. Ideally, the damage from the buffer holes should not exceed the damage zone from the perimeter holes as shown in Figure 6. In one of the blasts, all holes except the back holes were fired. If the buffer holes are charged spaced and burdened properly then the damage to the back can be minimized. As displayed in figure 7, the contour of the buffer row holes was pretty close to the design and the back holes were not damaged at all. ${ }^{9}$

\section{Perimeter holes}

Perimeter control blasting involves the control of drill hole spacing, burden and charge concentration near the contour of an excavation to provide a smooth and stable underground excavation. It is generally achieved by

\section{i. Reduced spacing between the blast holes}

ii. Burden, slightly higher than spacing, to facilitate the timely joining of the cracks between the contour holes

\section{iii. Loading blast holes with light decoupled charges}

Sometimes relief holes are drilled to promote crack formation between the loaded and unloaded holes Based upon the field tests and observations in operating mines, following approach is proposed to determine spacing, burden and explosive charge for perimeter holes.

Rock mass rating $(\mathrm{RMR})^{10}$ can be conveniently used for rock site classification because most of the mines have this information for geotechnical purposes.

Explosive charge per meter of charge length $\left(\mathrm{E}_{\mathrm{c}}\right)$ in $\mathrm{Kg} /$ meter can be estimated as follows:

$$
\mathbf{E}_{\mathbf{c}}=\mathbf{E}_{\mathbf{f}} \times \mathbf{d}^{2}
$$

Equation 1

Where $E_{f}$ is the explosive charge factor; $d$ is the diameter of the hole in meters

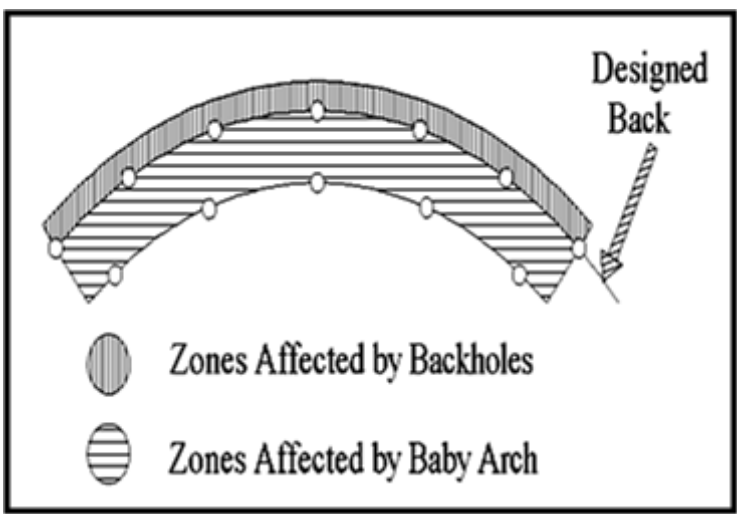

Figure 6 Damage from baby arch (buffer) and back holes

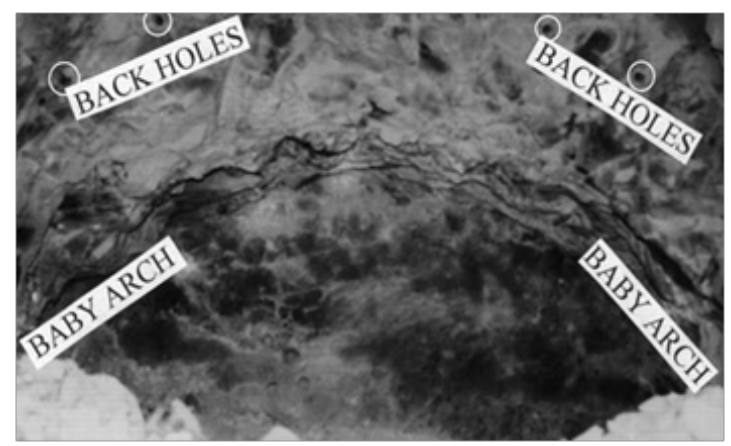

Figure 7 The contour of the excavation by firing up to baby arch (buffer) hole

\section{Spacing and burden between the holes}

The spacing and burden of perimeter holes have a significant influence on the shape and precision of an excavation. Higher spacing results in under break between the holes whereas too close spacing causes over break. The optimum spacing and burden for perimeter holes depends upon the rock type and the drill hole diameter.

$$
\mathbf{S}=\mathbf{S}_{\mathbf{f}} \times \mathbf{d} \quad \text { Equation } 2
$$




$$
\mathbf{B}=\mathbf{B}_{\mathbf{f}} \times \mathbf{S} \quad \text { Equation } 3
$$

Where $\mathrm{S}$ is the spacing between perimeter holes $(\mathrm{m}) ; \mathrm{S}_{\mathrm{f}}$ is the spacing factor; $\mathrm{B}$ is the burden for perimeter holes $(\mathrm{m}) ; \mathrm{B}_{\mathrm{f}}$ is the burden factor. Perimeter hole pattern design factors for different RMR values have been given in Table 1 .

\section{Delay time and sequencing of holes}

The simultaneous initiation of the back holes is important for the desirable formation of cracks along the row of perimeter holes. Crack lengths around the bore holes increase with the increase in the delay time. ${ }^{11}$ Normally, the back holes are fired with last or near last delay number which has a high scatter. The scatter in the firing time of the same number delays does not allow instantaneous initiation of perimeter holes. This problem can be overcome by the use of electronic detonators.

Table I Rock mass rating and perimeter hole pattern design factors

\section{Proposed Criterion for Blast Damage}

The strength and geology of a rock mass cannot be changed but their knowledge can facilitate the judicious selection of blast damage control measures. One can characterize the rock mass and formulate different strategies to excavate it with minimum damage. The rock mass rating (RMR) system developed ${ }^{10,12}$ was used in this study. The use of a single limiting value of Peak Particle Velocity (PPV), as in the case of blast damage criteria for residential structures, is not adequate and may result in damage to poor quality rock masses. Based upon the review of several case histories and work done by the author in different sites, the criterion for blast damage is proposed in Figure 8. The magnitudes of blast vibrations required for initiation and propagation of minor and major damage in rocks with different RMR values have been displayed. Minor damage includes loosening of open joints as well as joints with weak filling and reduction in the frictional properties of joints. Major damage is represented by fracturing of intact rock and rock falls. Based upon the quality of the rock mass, the blast design can be modified to keep the blast vibrations below the desired level.

\begin{tabular}{cccc}
\hline Rock mass rating (RMR value) & Spacing factor $\left(\mathbf{S}_{\mathrm{f}}\right)$ & Burden factor $\left(\mathrm{B}_{\mathrm{f}}\right)$ & Explosive factor $\left(\mathrm{E}_{\mathrm{f}}\right)$ \\
\hline $\mathbf{4} 45$ & $13-14$ & 1.25 & 75 \\
$\mathbf{4 5 - 5 5}$ & $14-15$ & 1.2 & 80 \\
$\mathbf{5 5 - 6 5}$ & $15-16$ & 1.15 & 90 \\
$\mathbf{6 5 - 7 5}$ & $16-17$ & 1.1 & 100 \\
$\mathbf{> 7 5}$ & $17-17.5$ & 1.05 & 105 \\
\hline
\end{tabular}

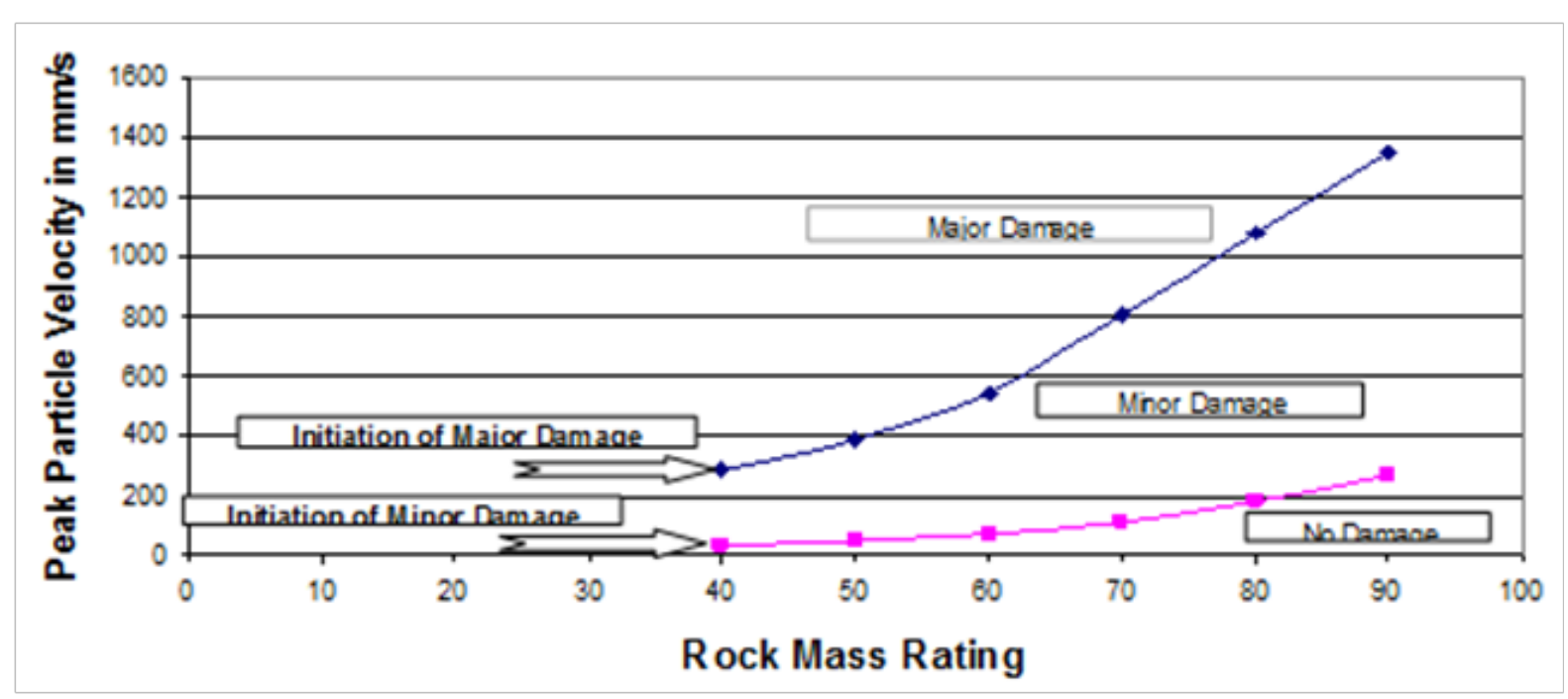

Figure 8 Magnitudes of blast vibrations for initiation and propagation of blast damage. 


\section{Conclusion}

Control measures to minimize the undesirable effects of blasting on the surrounding rock mass are crucial for the safety, economy and productivity of underground mines. Though each specific rock mass occurrence is unique yet there are common features in rock masses, which can influence the outcome of controlled blasting in a similar way. Blast damage control starts with the first hole to be marked and drilled. The key to the success of a blast damage control program lies in its acceptance by the drilling and blasting crew. Low damage in the case of high VOD explosives is partly attributed to the decoupling effect. Improperly designed and sequenced cut holes can produce significant damage to the contour of an excavation. If the hole spacing and charge concentration is optimum, initial cracks are produced between the neighbouring blast holes instead of around them. Based upon the quality of the rock mass, the blast design can be modified to keep the blast vibrations below the desired level. Proper planning, drilling accuracy and appropriate blast design can significantly contribute in achieving the goal of minimum damage, optimum productivity and safer working environment.

\section{Acknowledgments}

None.

\section{Conflict of interest}

Author declares that there is no conflict of interest.

\section{References}

1. Rathore SS, Bhandari S. Controlled fracture growth by blasting while protecting damages to remaining rock. Rock mechanics and rock engineering. 2007;40(3):317-326.

2. Mc Kown A. Perimeter control blasting for underground excavations in fractured and weathered rocks. Bulletin of the Association of engineering geologists. 1986;23(4):461-478.
3. Lewandowski T, Luan Mai, Danell R, et al. Influence of discontinuities on presplitting effectiveness. Proc. International symposium on rock fragmentation by blasting. 1996;217-225.

4. Singh SP. Blast damage control in jointed rock mass. Fragblast Journal. 2005;9(3):175-187.

5. Bauer GF, Donaldson DM. Perimeter control in development and breasting by use of a blasting program readily accepted by miners. Proc. 18th International conference on explosives and blasting techniques. 1992;133-141.

6. Liang WM, Liu H, Yang XL, at al. Effects of decoupled charge blasting on rock fragmentation efficiency. Proc. 12th ISRM International congress on rock mechanics. 2011:1237-1240.

7. Singh SP. Mechanism of tracer blasting. Geotechnical and Geological engineering. 1996;14(1):41-50.

8. Singh SP. Mechanism of cut blasting. Transactions of the institution of mining and metallurgy. 1995;104:A134-A138.

9. Singh SP, Xavier, Peter. Causes, impact and control of overbreak in underground excavations. Tunneling and underground space technology. 2005;20(2005):63-71.

10. Bieniawski ZT. Rock mechanics design in mining and tunneling. Balkema AA, editor. Netherlands; 1984.272 p.

11. Saiang D. Blast induced damage-a summary of SVEBEFO investigations. Technical report, department of civil and environmental engineering, Lulea University of technology. 2008;41.

12. Cunningham CVB, Goetzsche AF. The specifications of blast damage limitations in tunneling contracts. Tunneling and underground space technology. 1996;5(3):23-27. 